\title{
Characterizing the radio-X-ray connection in GRS 1915+105
}

\section{Prat* and J. Rodriguez}

DSM/IRFU/Service d'Astrophysique, CEA/Saclay, F-91191 Gif-sur-Yvette, France

E-mail: lionel.prat@cea.fr

\section{G. G. Pooley}

Astrophysics, Cavendish Laboratory, University of Cambridge CB3 OHE, UK

We analyzed radio (Ryle Telescope) and X-ray (RXTE, INTEGRAL) observations of GRS 1915+105, between May 1995 and June 2006, focusing on the times characterized by radio flares and cycles of hard dips - soft spikes in the X-ray lightcurve. Assuming these flares to be discrete ejections, we fitted a total of 687 radio flares with a standard model of a plasmon. We found that the distribution of width is $t_{0}=1160 \mathrm{~s}$ with an rms deviation of $360 \mathrm{~s}$, while that of the amplitude is $S_{\max }=59 \mathrm{mJy}$ with an rms deviation of $28 \mathrm{mJy}$. The distribution of width is thus rather peaked, while that of the amplitude not. Regarding radio and X-ray links, this study confirms previous observations on smaller datasets, namely that X-ray cycles of hard dips - soft spikes are always followed by radio flares. A strong correlation is found between the length of $\mathrm{X}$-ray "dips" in the X-ray lightcurves, and the amplitude and fluence of the subsequent radio oscillations. A model of an exponential rise of the form $L_{15 \mathrm{GHz}}(\Delta \mathrm{t})=L_{\max }\left(1-\exp \left(-\left(\Delta t-\Delta t_{\min }\right) / \tau\right)\right.$ is in good agreement with the observations, with the maximum fluence $L_{\max }$ of the order $70 \mathrm{Jy} . \mathrm{s}$, and the characteristic time $\tau$ of the order 200-500 s. We discuss possible physical interpretations of this correlation, regarding the physical process responsible for the ejection.

8th INTEGRAL Workshop - The Restless Gamma-ray Universe - Integral 2010

September 27-30, 2010

Dublin Ireland

*Speaker. 


\section{Introduction}

One of the main issues of Black-Hole Binary (BHBs) physics is the link between accretion and ejection processes. Indeed, if the strong gravitational potential favors naturally the accretion of matter, the occurrence of relativistic ejections of plasma in these systems is still far from being understood. In this respect, GRS $1915+105$ is a very promising object to conduct this study thanks to the unique wealth of behavior it displays (see, e.g., the review by [1]).

Contrary to transient microquasars, which are usually active for a few months between quiescent states, GRS 1915+105 has been constantly active for the 18 years since its discovery with GRANAT [2]. It shows a very high level of variability, which may be linked to the fact that GRS 1915+105 has a very high accretion rate compared with most other Low Mass X-ray Binaries (LMXBs). In particular, the X-ray lightcurves display cycles of hard dips - soft spikes specific to GRS $1915+105$. They are characterized by phases of low X-ray luminosity and hard spectra, which last between $\sim 10$ and $\sim 2000$ s (see Fig. 1 for sample lightcurves). These phases are ended by a short spike $(\sim 10 \mathrm{~s})$, which marks the end of the hard phase. Such features (hard X-ray dip ended by a spike) will be labeled simply "cycle" in the following. Usually, the light curve then shows a second spike, longer ( $\sim 100 \mathrm{~s})$, softer and brighter than the one marking the end of the hard dip. This second spike marks the beginning of a soft and highly variable phase, which ends when a new hard dip begins.

In the radio range, GRS $1915+105$ displays strong radio oscillations or isolated flares, which are clearly related to the X-ray dips [3, 4]. The power required to generate the synchrotron emission of these oscillations is likely to be a very significant fraction of the entire accretion energy of the system [5, 6]. Since X-ray cycles and radio oscillations seem to occur at the same times, it is tempting to look for a link between them and, thus, study both the X-ray and radio behaviors of GRS $1915+105$. The detailed results of our study can be found in [7], in this proceeding we will only present our main conclusions.

\section{A look at the radio flares}

We first searched for radio oscillations in the 11 years of Ryle Telescope data, and found about $\sim 1000$ clearly-identified flares. In order to study these flares, we used the widely known model described by [8], who calculated synchrotron emission from an adiabatically expanding cloud. In the case of GRS 1915+105, [4] observed delays in the lightcurves at different radio and infrared wavelengths compatible with this plasmon model.

In this model [8], flaring emission is produced through the adiabatic expansion of an initially optically thick blob of synchrotron-emitting relativistic electrons. During the first phase, the increase in the blob's surface area causes an increase in the flux. Then, the curve turns over as the plasma becomes optically thin because of the reduction in magnetic field, the adiabatic cooling of the electrons, and the reduced column density as the blob expands. Simultaneous observations at a lower frequency will show the same fractional rate of increase, but the maximum will be reached later and will have a smaller value. According to this model, the radio flux density scales as

$$
S_{V}(R)=S_{0}\left(\frac{v}{v_{0}}\right)^{5 / 2}\left(\frac{R}{R_{0}}\right)^{3} \frac{1-\exp (-\tau)}{1-\exp \left(-\tau_{0}\right)}
$$



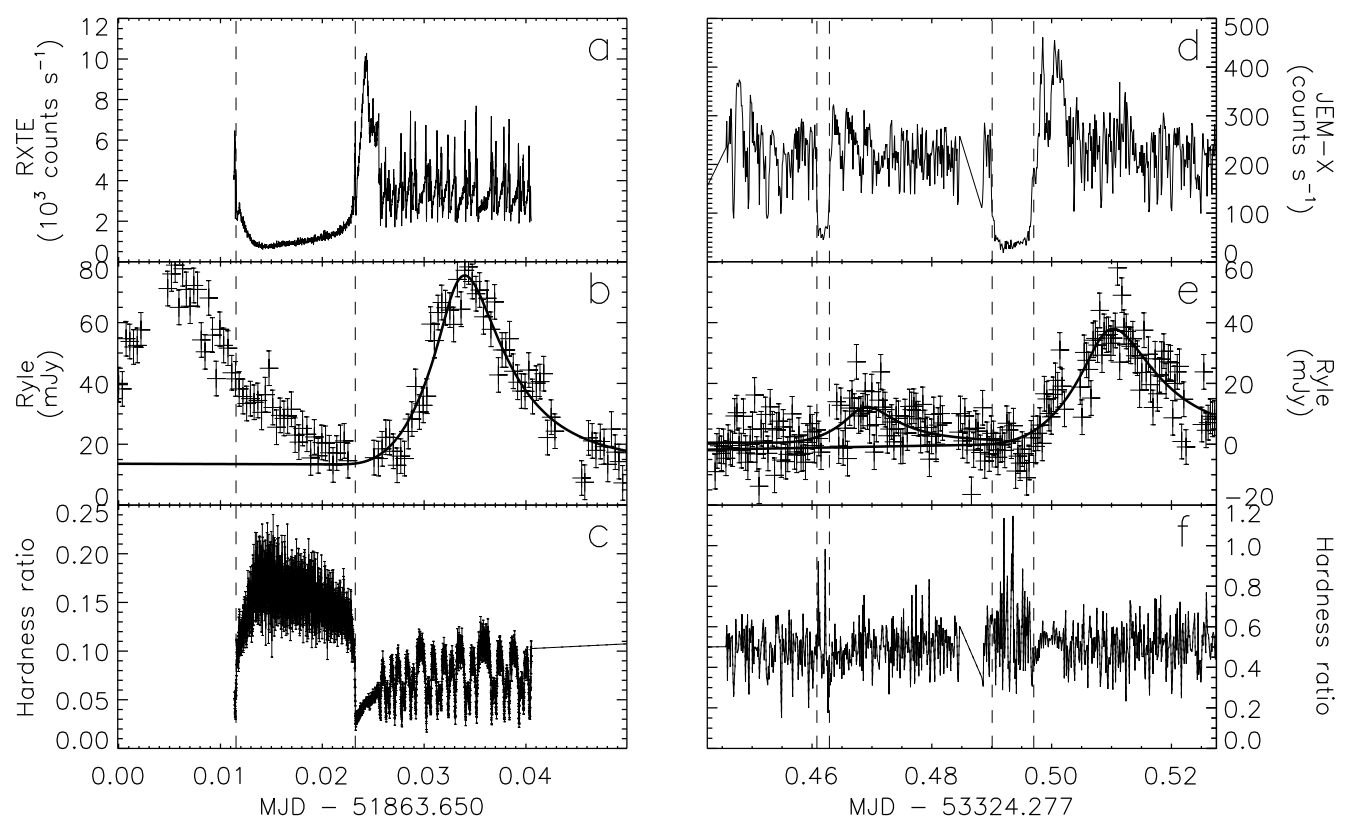

Figure 1: Simultaneous X-ray and Ryle observations of GRS $1915+105$. The plots show on the top the 2-60 keV RXTE lightcurve (panel (a)), and the 3-30 keV JEM-X lightcurve (panel (d)). Panels (b) and (e) display the $15 \mathrm{GHz}$ radio lightcurve, while panels (c) and (f) display the hardness ratios. The vertical dotted lines mark the beginning and the end of the X-ray dips on each panel. The continuous lines show the fit of the plasmon model on the radio data.

Here, $R, S, v$ and $\tau$ are the size, flux density, frequency and optical depth, respectively. We also assumed a linear expansion at constant speed $v_{\text {exp }}$ for the plasmon. Using this model, we fitted a total of 687 flares, and excluded very noisy data or incomplete flares. The distribution of flare width and amplitude appear to be gaussian. Gaussian fits to these histograms give a characteristic width of $t_{0}=1160 \mathrm{~s}$ with an rms deviation of $360 \mathrm{~s}$, and a mean amplitude of $S_{\max }=59$ mJy with an rms deviation of $28 \mathrm{mJy}$.

\section{Radio - X-ray links}

\subsection{X-ray dips and occurrence of radio flares}

In the X-ray range, we searched for RXTE and INTEGRAL data simultaneous with the Ryle Telescope pointings. A total of 54 hard X-ray cycles with radio coverage were found within these data (Fig. 1 shows two examples of such observations).

The analysis of these dips confirms the association between X-ray dips and radio flares: among the 54 X-ray dips, 52 are directly followed by a radio flare, only the 2 shortest dips being not followed by a detectable activity. Thus, we confirm that X-ray dips during the cyclic classes of GRS $1915+105$ (which are the $\alpha, \beta, \lambda$ and $\nu$ classes according to [9]) are always followed by radio flares.

The mean delay between the X-ray spike and the peak of the radio flare is $1040 \pm 185 \mathrm{~s}(1 \sigma$ standard deviation), a value consistent with the one calculated by [10]. As for the derived start time 

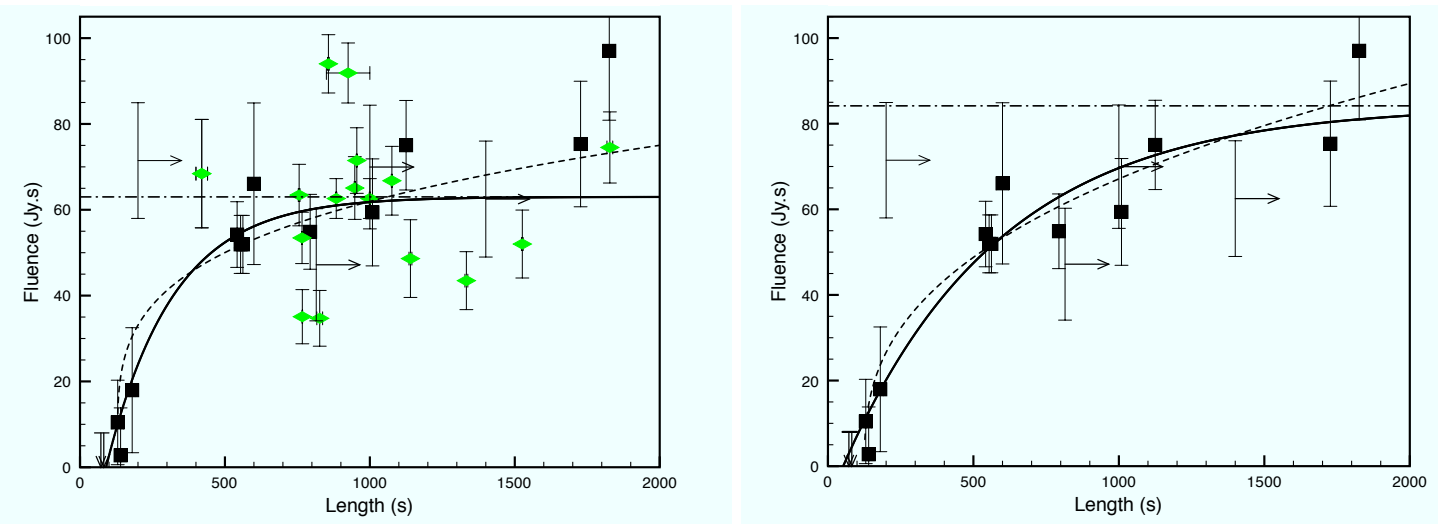

Figure 2: Fluence of the radio flares as a function of the dip-length $\Delta t$. The left panel displays all available observations, while the right one displays "isolated" radio flares only. The continuous line is an exponential fit and the dashed line a power-law fit to the data on each panel. The dot-dashed line marks the apparent saturation of the exponential in each case.

of the ejection, according to the van der Laan model, the ejection of matter is coincident with the time of the X-ray spike within less than 5 minutes. Thus, the X-ray spike can be safely identified with the trigger to the radio ejection.

\subsection{A correlation between duration of X-ray dips and fluence/amplitude?}

We then looked for correlations between the various parameters at hand. A pronounced trend is visible between the duration of the dips $\Delta t$ and the parameters of the subsequent flares: longer dips seem to be followed by more important radio ejections. In particular, a correlation is visible between $\Delta t$ and the fluence $L_{15 \mathrm{GHz}}$ of the following radio flare (Fig. 2).

To quantify this, we used the Spearman test of correlation, which detects any monotonic correlation in the data. This test shows a strong correlation between the length of the X-ray dip $\Delta t$ and the fluence of the following flare $L_{15 \mathrm{GHz}}$. Indeed, its value is $>99.99 \%$ in the case of "isolated" radio flares (flares for which the entirety of the flux can be associated to the ejection event), and 98.3\% using all available data, including flares detected on top of a strong radio background (radio flux which is probably not related to the radio flare event).

This correlation does not seem to be linear nor affine. Indeed, short X-ray dips produce much weaker radio flares than long dips (see Fig. 2). This is consistent with the statement of [11], who mentioned that dips shorter than 100 s do not seem to be followed by radio flares. Instead, the data are well described by either a power-law function or a (saturated) exponential function. Using a model of an exponential rise of the form $L_{15 \mathrm{GHz}}(\Delta \mathrm{t})=L_{\max }\left(1-\exp \left(-\left(\Delta t-\Delta t_{\min }\right) / \tau\right)\right.$, we obtain a maximum fluence $L_{\max }$ of the order 60-85 Jy.s, and a characteristic time $\tau$ of the order 200-500 s.

\section{Discussion}

Our analysis extends the previous results on the occurrence of radio flares $[11,10]$. With an extended data set compared to previous studies, we confirm that, during $\alpha, \beta, \lambda$ and $\nu$ classes, 


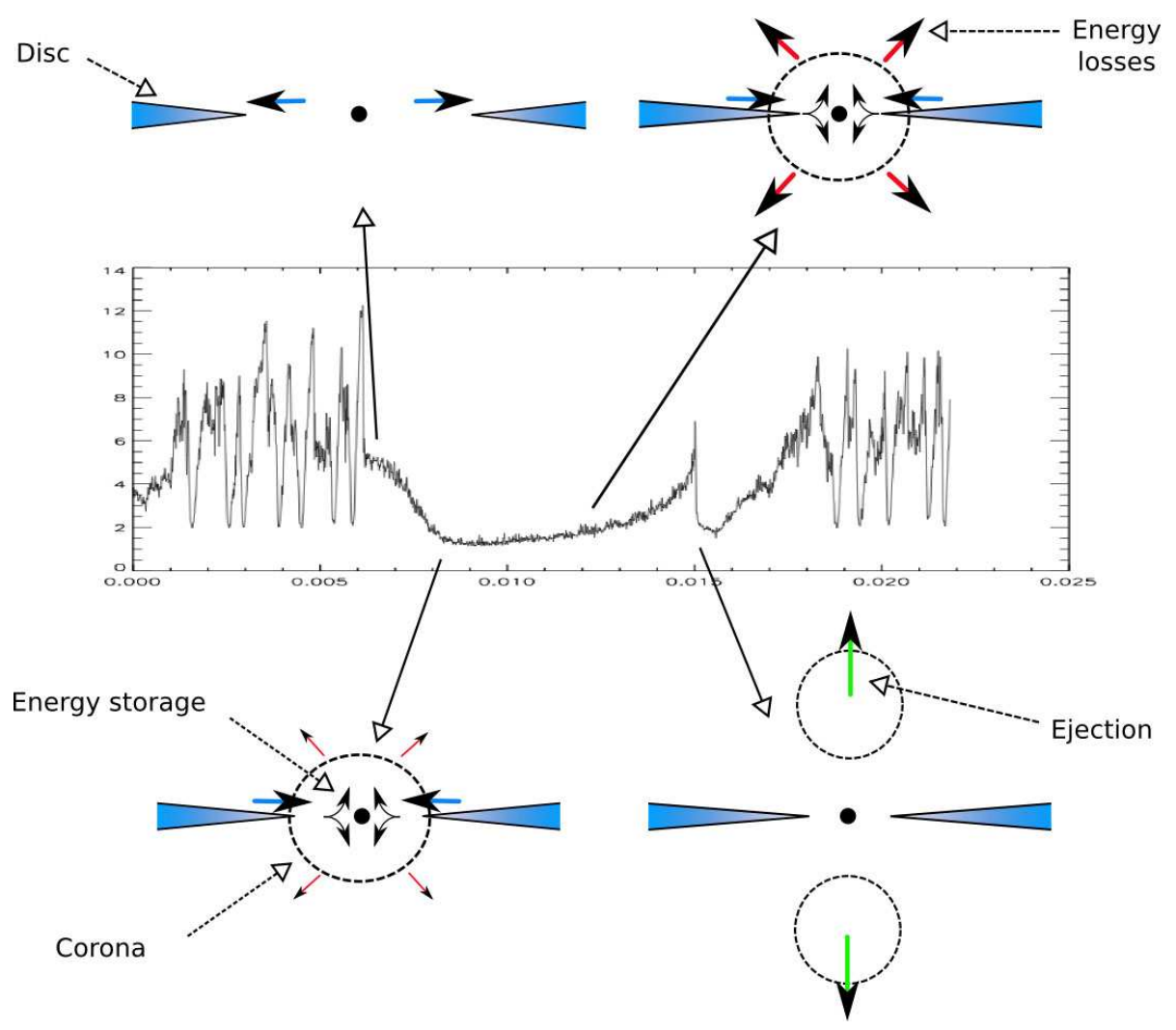

Figure 3: Sketch of the behavior of GRS 1915+105 during cyclic classes. At the beginning of an X-ray dip, the inner disc radius is high. During the disc itself, the disc gradually draws closer, bringing energy in. Part of this energy can be stored inside the corona, for instance in the form of magnetic energy. At the time of the final X-ray spike, the coronal material is quickly ejected from the system and becomes visible as a radio flare. Adapted from [12].

each and every $\mathrm{X}$-ray cycle in the lightcurve is followed by a radio flare. Moreover, the beginning of this flare coincides with the X-ray spike within less than 300s.

Using the available observations, a trend is visible between the length of the X-ray dips and the characteristics of the following flare: the longer the X-ray dip is, the bigger the following radio flare will be. Although the data are scarce, this link does not seem to be linear, and can be described, for instance, by a power-law or exponential relationship. Given the high uncertainties on the data, it is not possible to discriminate between these functions.

Yet, is we assume an exponential relationship, one could think of an attractive scenario to explain the correlation (Fig. 3). Observations show that the beginning of the dip is marked by a quick increase in the accretion disk inner radius. Then, during the dip itself, the disk draws closer to the $\mathrm{BH}[13,14,15]$. This evolution brings more gravitational energy close to the $\mathrm{BH}$. If we suppose that, during the Hard X-ray dip, energy is somehow extracted from the accretion disk and accumulated in the surrounding medium, the luminosity of the disk and corona will slowly increase. At the same time, the amount of energy lost by the corona in a given time grows as the energy density of the corona increases. Thus, the total energy stored inside the corona will saturates. This saturation is what is seen after $\sim 1000$ s. Then, at some point, this energy is released 
in the form of a quickly expanding blob of matter. In the X-ray lightcurve, this ejection is marked by a short X-ray spike $[4,10]$. The ejected material can come from the corona itself, or from the inner accreting disk; either way, after the spike the corona is not visible anymore, and the disk is closer to the $\mathrm{BH}$. Note that we tend to favor the coronal case $[15,7]$.

It is interesting to note that the distribution of width of the radio flares is quite peaked while that of the amplitude not. In the framework of the plasmon model [8], the width of the flare is related to the initial physical size of the plasmon. Thus, the relative sharpness of this distribution means that the initial radius of the plasmon is roughly always the same. It is tempting to interpret that this radius may be constrained by the inner radius of the disk. On the other hand, the maximum amplitude of the flare is related to the initial temperature and energy distribution of the ejected plasma. Thus, the relative broadness of the amplitude distribution could mean that the amount of energy stored inside the plasma prior to the ejection is more variable.

If we assume that the amount of energy stored inside the plasma depends on the duration of the preceding X-ray dip, then this relative broadness can be easily explained. Indeed, during shorter dips the input of energy would be shorter, thus the amplitude of the following flare would be smaller. Since the duration of the X-ray dips is highly variable, the amplitude of the flares will also be variable. On the other hand, the medium which stores this energy will always be located in the same region, close to the inner radius of the disk; if its physical size is constrained by the disk, then the width of the flares will be roughly constant.

\section{References}

[1] Fender R., Belloni T., 2004, ARA\&A, 42, 317

[2] Castro-Tirado A. J., Brandt S., Lund N., 1992, IAU Circ., 5590, 2

[3] Pooley G. G., Fender R. P., 1997, MNRAS, 292, 925

[4] Mirabel I. F., Dhawan V., Chaty S., Rodriguez L. F., Marti J., et al. 1998, A\&A, 330, L9

[5] Fender R. P., Pooley G. G., 2000, MNRAS, 318, L1

[6] Meier D. L., 2001, ApJ, 548, L9

[7] Prat, L., Rodriguez, J. \& Pooley, G. G. 2010, ApJ, 717, 1222

[8] van der Laan H., 1966, Nature, 211, 1131

[9] Belloni T., Klein-Wolt M., Méndez M., van der Klis M., van Paradijs J., 2000, A\&A, 355, 271

[10] Rodriguez J., Hannikainen D. C., Shaw S. E., Pooley G., Corbel S., et al. 2008a, ApJ, 675, 1436

[11] Klein-Wolt M., Fender R. P., Pooley G. G., Belloni T., Migliari S., Morgan E. H., van der Klis M., 2002, MNRAS, 331, 745

[12] Prat, L., 2010, PhD Thesis

[13] Belloni T., Mendez M., King A. R., van der Klis M., van Paradijs J., 1997, ApJ, 488, L109

[14] Migliari S., Belloni T., 2003, A\&A, 404, 283

[15] Rodriguez J., Shaw S. E., Hannikainen D. C., Belloni T., Corbel S., et al. 2008b, ApJ, 675, 1449 